Cahiers $d u$ MONDE RUSSE

\section{Cahiers du monde russe}

Russie - Empire russe - Union soviétique et États indépendants

$50 / 2-3 \mid 2009$

L'Europe orientale, 1650-1730. Crises, conflits et renouveau

\title{
The Musical Comedy Films of Grigorii AleksandrovRimgaila SALYS
}

, Bristol - Chicago : Intellect, The University of Chicago Press, 2009, 352 p. (nombreuses photographies)

\section{Cloé Drieu}

\section{(2) OpenEdition}

\section{Journals}

Édition électronique

URL : https://journals.openedition.org/monderusse/9761

DOI : 10.4000/monderusse. 9761

ISSN : $1777-5388$

Éditeur

Éditions de l'EHESS

Édition imprimée

Date de publication : 15 septembre 2009

ISBN : 978-2-7132-2260-3

ISSN : $1252-6576$

Référence électronique

Cloé Drieu, "The Musical Comedy Films of Grigorii AleksandrovRimgaila SALYS », Cahiers du monde russe [En ligne], 50/2-3 | 2009, mis en ligne le 14 janvier 2013, consulté le 02 septembre 2022. URL http://journals.openedition.org/monderusse/9761 ; DOI : https://doi.org/10.4000/monderusse. 9761

Ce document a été généré automatiquement le 2 septembre 2022

Tous droits réservés 


\title{
The Musical Comedy Films of Grigorii AleksandrovRimgaila SALYS
}

\author{
, Bristol - Chicago : Intellect, The University of Chicago Press, 2009, \\ $352 \mathrm{p}$. (nombreuses photographies)
}

\section{Cloé Drieu}

\section{Rimgaila SALYS, The Musical Comedy Films of Grigorii Aleksandrov, Laughing Matters, Bristol - Chicago : Intellect, The University of Chicago Press, 2009, 352 p. (nombreuses photographies)}

1 Se fondant sur des sources publiées (presse essentiellement), des sources d'archives (Gosfilmofond, RGALI) et des témoignages ou mémoires ${ }^{24}$, l'auteur de ce livre consacre un chapitre à chacune des quatre grandes comédies musicales à succès réalisées par G. Aleksandrov (1903-1983) : Veselye rebjata [Les Joyeux Garçons] (1934), Cirk [Le Cirque] (1936), Volga Volga (1938) et Svetlyj put' [La Voie lumineuse] (1940). Le déroulement chronologique est ainsi privilégié, ce qui permet, grâce à une analyse du contexte culturel et politique de production de ces films (des premières ébauches de scenarii à la création cinématographique), de mettre au jour l'évolution des représentations - d'un premier film très marqué par la NEP et réalisé dans un style « revue-lutionnaire » (Veselye rebjata) à la consécration musicale du réalisme socialiste à la veille de la Grande Guerre patriotique (Svetlyj put'), en passant par la mise en image de l'amitié des peuples (Cirk) ou de Moscou comme centre unificateur (Volga Volga).

2 Proche collaborateur de S. Eisenstein (acteur et assistant-réalisateur pour La Grève et Le Cuirassé Potemkine, co-scénariste pour Octobre et L'Ancien et le nouveau), Aleksandrov passe trois années aux États-Unis (Hollywood), où il se lie d'amitié avec Chaplin et fréquente les studios Disney. Ceci constitue pour lui une expérience fondatrice dont il tire un large bénéfice à son retour en Russie, en mai 1932, alors que l'activité cinématographique connaît de profonds bouleversements liés au processus de centralisation et de reprise en main des activités artistiques par le pouvoir politique, caractéristiques de la période. C'est précisément cette expérience américaine que Rimgaila Salys tend à remettre au 
centre de l'analyse de la création cinématographique d'Aleksandrov en insistant sur l'influence, entre autres, du musical américain et de la tradition comique qui lui est associée. Ainsi, pour chacun des films, sont étudiés avec beaucoup de minutie les emprunts et détournements de certaines scènes de comédies musicales ou de films américains (Chaplin, Lloyd, Keaton, Marx Brothers), de dessins animés de Disney (la part d'animation dans les films d'Aleksandrov est à souligner) ou du surréalisme de Buñuel (dans Veselye rebjata notamment). On pourrait aussi soulever la question, qui n'a pas été posée, des emprunts inverses: Chaplin n'aurait-il pas pu être influencé par l'une des premières scènes de Cirk lorsqu'il représente Adenoid Hynkel, dans Le Dictateur (1940), jouant avec un ballon représentant le globe terrestre? Cet aspect de l'étude permet d'insister sur la porosité de la cinématographie soviétique aux influences étrangères, et ce jusqu'à la veille de la Seconde Guerre mondiale, malgré la diminution importante de la part des films étrangers distribués dans les salles d'URSS à partir de 1929.

Pourtant, si ces comédies musicales de l'ère stalinienne - joviales, pleines d'entrain et emblématiques des paradoxes de cette période - conservent sans les dénaturer, dans un premier temps, les grands paradigmes du genre de la comédie musicale américaine, elles intègrent progressivement les traits du réalisme socialiste. L'analyse de ces quatre films met en évidence l'évolution des valeurs socioculturelles de référence durant le premier stalinisme : les relations sociales ou nationales, fraternelles, horizontales et égalitaires jusqu'au début des années 1930 laissent place à une représentation hiérarchisée et verticale, où le parti devient bien évidemment le pivot central de toute relation. À ce titre, les rapports de genre (et de couple) et le langage du corps font l'objet de considérations particulières de la part de l'auteur qui insiste sur un éloignement physique croissant, la domestication des sentiments et des gestuelles (retenue, mesure, maitrise) et, finalement, sur une relation homme-femme totalement dépassionnée qui ne peut se réaliser qu'une fois les objectifs (de production, de travail) atteints (Svetlyj put'). De fait, l'étude de l'intertextualité des œuvres change progressivement de registre: si celle-ci se lisait d'abord dans la jovialité et son rapport à la cinématographie américaine surtout (Veselye rebjata, Cirk), elle se recentre ensuite sur un monde soviétique de plus en plus tendu, qu'il soit artistique (satire de Meyerhold dans Volga Volga par exemple), politique (références discrètes au climat de répression des années 1930 dans ce même film) ou idéologique (Svetlyj put', qui présente un code particulièrement abouti des valeurs socialistes).

4 L'étude de l'auteur fait aussi la part belle aux continuités sur la longue durée : reprise de grands traits de la littérature russe (critiques des bureaucrates, héros socialistes de type aryen réinvestissant la figure du bogatyr', protecteur et courageux), de la mythologie, ou d'autres classiques de la littérature (Cendrillon de Perrault par exemple). La musique et les chants offrent un terrain particulièrement propice à l'analyse de cet aspect puisque, en réinvestissant les mélodies ou les chansons du folklore russe, ils valorisent non seulement un genre mineur face à une culture d'élite qui relève du répertoire classique, mais ils légitiment surtout le pouvoir soviétique en favorisant son appropriation par l'audience. Les mélodies s'inspirent du passé alors que les paroles louent le nouveau pouvoir et se révèlent comme une force unificatrice sans pareil et un vecteur d'identification et d'appartenance à une même communauté de destin, processus dont la portée est indéniable à en croire le succès des films en salle. Celui-ci est parfois faramineux (40 millions de spectateurs pour Cirk entre 1936 et 1939), et la consécration du public se double de celle du pouvoir: les principaux et meilleurs artistes du moment (paroliers, compositeurs, chefs opérateurs, réalisateurs, acteurs et actrices) sont parfois 
décorés par la plus haute autorité soviétique et reçoivent, pour certains, le prestigieux Prix Staline. Mais ce succès doit aussi être évalué en fonction de l'impact des chants sur la société et de leur réutilisation lors des grandes célébrations politiques, du fait notamment d'importants tirages en vinyle qui permettent aux chansons de dépasser le cadre strictement cinématographique. «La marche de Veselye rebjata » est reprise lors de la grande parade du $1^{\text {er }}$ mai 1935. Mais surtout, c'est «La chanson de la mère-patrie » (Cirk) - dont les paroles font directement écho à la Constitution de 1936 - qui va devenir un « deuxième hymne national » et la musique officielle de toute célébration relative à la Loi fondamentale : elle ouvre les programmes de radio, serait chantée par les soldats partant au combat et même utilisée par les partisans pour vérifier l'identité des parachutistes tombés sur leur territoire. "Je ne connais aucun autre pays où l'homme peut respirer si librement » reprend en chœur, à la veille des purges de 1937, Ljubov' Orlova, égérie et épouse d'Aleksandrov, dans le rôle de l'Américaine Marion Dixon, alors que celle-ci trouve définitivement refuge en Union soviétique après avoir fui les ÉtatsUnis où elle risque le lynchage pour avoir accouché d'un enfant métis (Cirk).

Ce chant en particulier, ou encore le voyage tout le long du canal de la Volga, qui est ouvert en juillet 1937 (Volga Volga) et réunit l'ensemble de la partie européenne de la Russie, participent de la célébration de l'unification des périphéries au centre. Aleksandrov l'affirme lui-même : le rôle du cinéaste est bien de montrer l'étendue et la diversité du pays afin que les citoyens soviétiques puissent connaître leur patrie. Si le thème de l'intégration d'une communauté multiethnique et multinationale est si important, il aurait bien sûr été intéressant d'analyser la réception d'un film comme Cirk - qui insiste sur l'«amitié des peuples », l'acceptation de tous quelles que soient les différences physiques et raciales, la représentation d'une grande famille pluriethnique et plurilinguistique - auprès d'un public non russophone. Si le russe est une langue unificatrice par excellence, sa connaissance n'est pourtant pas indispensable à cette grande communion soviétique qui repose sur des valeurs d'assimilation, d'égalité ou d'idéaux supérieurs: la nouvelle "Marija Dikson" parle très peu russe, ce qui ne constitue jamais un obstacle à son intégration. Évidemment, il aurait aussi été intéressant de décrypter la portée des chants dans le processus de russification, façon ludique et entrainante de véhiculer un message patriotique et de défense du territoire face à l'ennemi. De même, nombre de situations comiques reposent sur un humour gestuel et des gags de nature visuelle - au détriment de gags verbaux - proches de la culture populaire ou de la pantomime, ce qui facilite une compréhension universelle et transcende la cacophonie soviétique des langues.

Si ce travail est très précieux par la minutie des analyses et le souci du détail, on lui reprochera de ne pas avoir suffisamment évoqué, de façon transversale, les grandes thématiques développées pour chacun des films. À ce titre, l'auteur n'a apparemment pas jugé nécessaire de conclure son ouvrage, ce qui aurait pourtant permis de conceptualiser davantage l'ensemble. On pourrait également lui reprocher de ne pas avoir suffisamment éclairé les questions purement techniques de prise de son, de synchronisation, etc. Ces quelques remarques n'enlèvent cependant rien à la qualité de l'ouvrage qui, par de nombreux aspects, est très stimulant pour des recherches tant sur la cinématographie soviétique que sur les rapports entre art et politique. 


\section{NOTES}

24. À commencer par ceux d'Aleksandrov lui-même: Gody poiskov i truda [Les années de recherches et travaux] (1975) et Epoha i kino [Une époque et le cinéma] (1976). 